\title{
Disaster Network Science: Research and Applications
}

\author{
Liaquat Hossain $^{1 *}$ and Shihui Feng ${ }^{2}$ \\ 1 Library and Information Management Program, Division of Information and Technology Studies, The University of \\ Hong Kong, Hong Kong, China, IInformation Management, Division of Information and Technology Studies, \\ The University of Hong Kong, Hong Kong, China
}

Keywords: disaster, network science, trans-disciplinary, systems approach, communication

\section{INTRODUCTION}

In an increasingly complex, mobile, and interconnected world, we face increasing threats of disasters, whether by chance or deliberately. Disruption of coordinated response and recovery efforts due to organizational, technical, procedural, random, or deliberate attack could result in the risk of massive loss of life. For example, extreme public concern over pandemics during the past decade suggests that urgent action is required to develop optimal information-sharing environments for promoting good health (Heymann, 2004; Shapiro et al., 2010). Innovative approaches to information flow modeling and analysis for dealing with challenges of coordinating across multilayered agency structures and development of early warnings through social systems using social media analytics may be pivotal to timely response to global disasters.

Internet is seen as a critical medium that diffuses information not only through announcements

OPEN ACCESS

Edited by:

Natalie Danielle Baker,

Virginia Commonwealth University,

USA

Reviewed by:

Spyridon Samonas,

Louisiana Tech University, USA

*Correspondence:

Liaquat Hossain

Ihossain@hku.hk

Specialty section:

This article was submitted to Disaster

Communications,

a section of the journal

Frontiers in Communication

Received: 24 December 2015

Accepted: 25 April 2016

Published: 10 May 2016

Citation:

Hossain L and Feng S (2016) Disaster Network Science: Research and Applications.

Front. Commun. 1:1.

doi: 10.3389/fcomm.2016.00001 by government agencies but also through informal channels, ranging from press reports to media, such as blogs and twitter showing interplay of social activity and human mobility in a large realworld population (Sadilek et al., 2012). Increasingly, public sources provide new ways to create timely warnings and prediction of global disasters (i.e., disease outbreaks detection through search query behavior), which can be achieved by creating a computational platform to capture community-based interactions data through the usage of social media (Ginsberg et al., 2009). Moreover, the use of informal electronic information has been credited with reducing time to detection of epidemics and facilitates timely public health responses (Brownstein et al., 2009). Network science approach provides us with opportunity to develop complementary large-scale abstract modeling and individual and community meaning making to create more useful and sensitive disaster preparedness and response research.

\section{DISASTER NETWORK SCIENCE}

Network science is an emerging area of research, which relies on the theoretical and empirical basis of social sciences, information analytics, and computational and statistical mechanics to infer meaning from large volume of data from different sources (Thomas and Wong, 2004; Watts, 2004). Graph theory is regarded as the mathematic foundation of network science, which can trace back to the study on Königsberg Bridge in 1736. In the early 1930s, social network analysis started with a focus on studying the interactions among social actors. In 1950s, Bavelas (1950) pointed out that the communication structures have influence on efficiency of information diffusion, and centrality positions of actors have impact on the influence of positions. In 1973, Granovetter (1973) proposed the theory of tie strengths suggesting weak ties are more efficient for people to acquire new information. Since 1990s, with the development of studies on network theories and the improvement of computing, complex networks have emerged as a new advancement of network science. Complex networks are 
constructed by irregular, complex, and dynamic structures focusing on studying the evolution of large-scale networks and properties of dynamical units (Boccaletti et al., 2006). Complex network provides a better way for us to conceptualize the characteristics of real network and analyze the dynamic evolution of large-scale networks, which can be used to characterize the behavior and study the optimal functioning of hierarchical and social organizing systems for disaster preparedness and response. Network science integrates methods and analytical techniques drawn from mathematical sociology (i.e., social networks analysis), social anthropology, and computer science (i.e., information visualization, graph theoretic approaches, and data mining techniques, such as clustering) from large social, organizational, and learning interactions data. It offers opportunity to recognize information needs and identify, evaluate, and use information effectively with the possibility to develop the capacity for future social sciences research confronted with the challenges in dealing with large volumes of data and uncertainty (Bruce, 1999; Eisenberg et al., 2004; Barabási, 2007).

Disaster network science can be conceptualized as (i) modeling of dynamic flow of information at multilevel, (ii) robustness of the flow of information across multilayered information-sharing environment, and (iii) efficiency of the network structure for communication flow, which are regarded as key functional properties of disaster preparedness and response systems. The underlying theoretical foundations for exploring networks in a globally interconnected society drawn from sociology, social psychology, computational methods, graph theory, and statistical physics can demonstrates its application in a wider local and global societal context. Network science as a theoretical and analytical approach can support development for new theories and develop algorithms to detect complex shared and overlapping social and organizational network structures that are critical to develop robustness for disaster preparedness and response research. It will aid in the development of a new methodological base for studying dynamics of complex networks and develop tools for modeling and analysis of large complex dynamical network structures, which have impact in investigating a wide variety of phenomenon [i.e., social structures and individuals and group work, financial market systems (crisis), modeling disaster/crisis response in large-scale tsunami, fire, and spread of diseases, such as Ebola, as well as modeling emerging community networks using social media]. For example, issues for mobile computing in a variable work context suggest that an emergency field health-care worker can benefit from the development of user and experience-centered design in HCI for supporting delivery of timely and effective interventions to community (York and Pendharkar, 2004).

Key research questions that can guide disaster network science research are - how can hierarchical coordinating systems combined with the knowledge of community-based emerging ad hoc networks help support digital interventions for effective and efficient preparedness and response to challenges that we face for future emergencies, health, and well-being? This requires not only understanding the existing multijurisdictional formal coordinating structures but also accessing and sharing of locally situated knowledge through digital interventions and cultivation of community-based ad hoc networks. It can further suggest design of prototype interventions to be tested in lab and field (around, e.g., visualization, participatory social media platform design, educational apps, etc.) using big data, use of that data in local communities and globally, community participation in design of interventions.

\section{DISASTER RESILIENCE}

Resilience may take different forms in different systems. For the spread of epidemics, it may be important to be able to cut connectivity and isolate infected areas. Modular structure has been observed during disasters and mass casualties to provide resilience to changing conditions, by enhancing adaptability (Born et al., 2007). Yet, it may impede critical information flow in crisis situations and reduce resilience to black swan events (Taleb, 2005). The integration of network science to the study of disaster and resilience can transform our knowledge of what makes a system resilient to diverse changing circumstances in relation to digital interventions to support well-being. For example, Lindley et al. (2009) from Microsoft Research Lab at Cambridge experimental study on user-centered design in human-computer interaction (HCI) for exploring resilience of household trials with BubbleBoard was designed to create an improved answer machine and to encourage family members to appropriate it through the inclusion of open and playful design elements. Leveraging the theoretical and modeling strengths of network science, disaster research can develop and test new theory of how systems collapse, how to detect and avert such collapse for social and organizational systems, which display strong resilience, and incorporate the findings into new methods for building and maintaining resilience. One of the major challenges is to incorporate user and experience-centered design for making the social and organizational system more adaptive and resilient. HCI challenges for developing community-based disaster recovery have also been considered an emerging and important area within recent initiatives under digital human modeling research and practice (Streefkerk et al., 2014). To address these issues of resilience, at both a fundamental and applied level, disaster preparedness and response research needs increasing focus on the followings:

- Theory (Network Science) a trans-disciplinary systems approach, bridging theoretical and methodological gaps through integration of social, organizational, computational, and information sciences and its application for modeling social and organizational networks at a local and global level in developing human-centered design (HCD) of digital interventions for emergencies, health, and well-being;

- HCD to drive a commitment to HCD of digital interventions by developing social recommender systems for communities, organizations, and further development of test bed for justin-time public education, particularly in emergencies, health, and well-being;

- Big Data to study social and organizational systems (i.e., both open and closed systems), such as community participatory networks, formal hierarchical organizational networks, 
informal communications with multijurisdictional organizational networks, and to apply and refine the theoretical framework within the context of emergencies, health, and well-being; and

- Computation (Social and Organizational Networks Modeling, Simulations, Social Media Analytics, and other methods) to combine all the above for testing HCD of digital interventions for emergencies, and other areas such as (i) social media based early warning using search query trends for infectious diseases, (ii) use informal networks to create experience and meaning for facilitating shared learning, and (iii) targeted approaches for Government and community-based organizations to use digital interventions by capitalizing on open social media-based big data platform for improving their services to support future emergencies.

\section{OPERATIONALIZING DISASTER NETWORK SCIENCE}

Previous studies have also shown that design of certain types of network aid in supporting improved performance better than others within disaster context (Hossain and Kuti, 2010). Network science approach for disaster research and practice can support development, empirical validation, and use computational models and theories by investigating the whole system-level resilience of social and organizational systems and their interactions at micro- and macrolevel using both static and dynamic modeling of networks. Network modeling of disaster preparedness and response also offers opportunity to verify the developed models by characterizing and visualizing disaster networks using existing national data from Federal Departments as well as quantitative and action-based observational research for field studies. In doing so, network science in disaster research and practice can provide a fundamental theoretical and empirical basis for crossfertilization of resilience models across social, organizational networks, and computational science. These can be integrated further in a single framework that allows forecasts to the study of adaptive and self-organizing network dynamics of social and organizational systems under stress. Such an approach linked to dynamical systems modeling to correlate network structure to behavior of information flow over time will be significantly more robust. This will allow us to discover how changes in actors' and organization's decision-making rules, interconnections among actors and organization, and fitness function that actors and organizations employ will produce different aggregate outcomes during and aftermath of disasters. Below, we present five such taxonomies for disaster preparedness and response using network science.

\section{Multilayered Knowledge Bases}

Multilayered knowledge bases that will integrate knowledge areas across the agencies involved in coordinated responses to disasters and provide empirical validation for this knowledge base using the targeted disaster scenarios. The effectiveness of an explicit, shared knowledge base depends on far more than explicit designations, status, and roles of personnel: the size and relevance of the associated tacit shared knowledge bases are crucial. Directly related to this will be the research question of whether the coupling of the explicit and the tacit allows for more effective coordinated responses to disasters, which requires multijurisdictional efforts.

\section{Coordinated Learning Networks}

Coordinated learning networks develop the structures that capture the evolution and adaptation of coordinated responses, which will effectively and efficiently detect node failures, by simultaneously and rapidly converging both formal hierarchical and informal self-organized structures to an optimal dynamical response, within the context of changing circumstances. Using these learning networks in the test cases, identification and evaluation of existing communication structures among organizations, states, systems, and processes within existing disaster responses network can be explored for optimal functioning. Thus, quantify the converging information flow, relate the network structure to disaster responses, and identify bottlenecks, all in the context of rapidly changing conditions in the field. This new understanding will be an essential quantitative underpinning of "lessons-learned activities" and will deliver optimal responses in real time. Using systems approach to study formal and informal communications of authorities at a local, state, and federal level and other multijurisdictional coordinating agencies responsible for disaster preparedness and response programs and, in particular, emergency management, disease management for foodborne, zoonotic, and other human and bio-related disaster medicine and public health preparedness.

\section{Capability and Risk Assessment Model}

This capability and risk framework is a necessary underpinning, which can facilitate the development for organizational capability and risk assessment, critical to disaster preparedness and response. It can demonstrate preparedness and risk factors for organizations dealing with disasters. Further attempts can be made to test the generalizability within the context of a wider disaster response. This can support developing computational models for disaster risk trajectory analysis and design digital interventions through experience-centered design to promote and provide empirical basis for evaluating disaster preparedness and response through community participatory networks and user-centered design.

\section{Agency and Generalized Behavior Models}

This challenging development dealing with two very different layers of response systems, i.e., types of organizations, which are both hierarchical command control driven and local and community based have not been previously attempted. One is more procedural and hierarchical command driven guided by formal standard operating protocol, the other is motivated by informal and self-organized community-based volunteer-based organizations, structured quite differently, in terms of their operational procedure. The real challenge is to develop the conceptual foundations, which will capture these elements of operations in a single converging model. The integration of qualitative and quantitative social networks may help generate new insights as to the functioning of disaster responses. It can complement the 
quantitative social networks by adding richer description and analysis of the rationale for such functioning.

\section{Network-Enabled Coordination}

Network-enabled coordination increased efficiency in improving hierarchical and ad hoc organizational information flow and bottlenecks can be designed and tested for dealing with disasters. It can provide validation for using social networks in exploring the optimal functioning of disaster responses. This solution can be a significant advance in the knowledge base required to develop similar outcomes for the management of disasters, such as terrorist threats, bushfire threats, massive power outages, tsunamis, floods, and other public response that involves swift, multilayered coordination. This may produce insightful models that will give better understanding of social and organizational processes in disaster preparedness and crisis management with a view to inform the design of interventions, including sociotechnical, policy, etc., at a local and global level. Using social networks and computational modeling combined with organizational and behavioral theory to develop capacity for addressing the growing area of research in disaster preparedness and response leveraging big data with a focus on visualization, usability, and community participation for preparedness and early detection to emergencies, health, and well-being indicators. The outcome would facilitate shared understanding of risks for disaster preparedness of global burden and epidemics. This can be achieved by creating a platform to link seemingly disparate data sets across widely different disciplines, and further provide the means to interpret these data in terms of the complex interdependencies between environment, society, and health and well-being.

\section{IMPLICATIONS OF DISASTER NETWORK SCIENCE}

\section{Digital Interventions through $\mathrm{HCl}$ for Public Health Disasters}

Public health problems are usually attributed to individual behaviors, such as inadequate diet, absence of exercise, smoking, drinking habits, and access to addictive substances. Such attribution is clearly overly simplistic. The behavioral network dynamic perspective can be used to explore the inter-connectedness of nutrition, epidemiology, and immunity. The effects of behavioral network variables, such as tie strengths (i.e., strong ties and weak ties), and their joint effect on digital interventions to health education can provide a comprehensive synthesis demonstrating the importance of behavioral network dynamic theories for improving interventions in dealing with health and well-being. Therefore, it is important to recognize the importance of external influences on health and health-related behavior.

An alternative approach acknowledges the shifting importance of the various major factors of health at different stages of the lifespan, the powerful early influence of primary socialization being one example. Although this view focuses more on individuals and their cumulative experience rather than a more general overview of determinants, there are an increasing number of research studies suggesting that social relationships and social support play an important role in the health scenario. The role social multiplier effect has influence on lifestyle choice could lead to more effective intervention by promoting pre-existing relationships and new social influences that promote positive influences. The conceptual model developed by Berkman et al. (2000) also identifies four primary pathways: (i) social support, (ii) social influence, (iii) social engagement and attachment, and (iv) access to resources and material goods through which social networks operate at the behavioral level.

Social networks theory and models have been extensively applied in many different scenarios (Borgatti et al., 2009). For example, network theories can provide insights into "measuring affiliation to check if two people with the same affiliation know each other, and hence, are more likely to spend more time together than two people picked at random" (Wasserman and Faust, 1994) or to analyze social structures based on location and proximity to confirm if people follow patterns of movement and joint purpose, and, consequently, to predict their movements (Carrington et al., 2005). Furthermore, drawing up social networks also allows us to define people's status relative to the information flow in the social network (Marsden and Friedkin, 1993). A multitheoretical approach is proposed here in order to develop a robust behavioral network dynamics required to support the arguments about the importance of the ties and influence ego networks. Theories of sociology (Turner, 1988) and social psychology (Wish et al., 1976), such as social capital (Burt, 2000), strong and weak ties theories (Granovetter, 1973; Krackhardt, 1992), social influence (Friedkin, 1998), social learning (Bandura, 1978), personal networks (Wellman, 1988) and tie strengths (Krackhardt, 1992), can provide evidence of the mechanisms that propel, sustain, and dissolve interactions among members of group, community, family, and close friends (Pentland et al., 2005). Therefore, those theories support a better understanding and visualization of how dietary habits, physical activities, information exchange, and support can be explored, learned, spread, and integrated into the daily life of children and their respective personal networks (Hanish and Rodkin, 2007). We aim to deliver digital interventions for prevention and treatment of disease and disability by incorporating HCI to promote digital interventions for online social support and to develop family and community strengths and abilities for effective health interventions. For example, Kalichman et al. (2003) preliminary results from a community survey for health-related Internet use, coping, social support, and health indicators in people living with HIV/AIDS provides evidence of integrating user and experiencecentered design in HCI for support health and well-being.

\section{Social Networks, $\mathrm{HCl}$, and Computation for Global Disease Epidemics}

In an increasingly complex, mobile, and interconnected world, we face increasing threats of invasive diseases, whether by chance or deliberately. Disruption of coordinated response and recovery efforts due to organizational, technical, procedural, random, or deliberate attack could result in the risk of massive loss of life. There has been significant effort within the HCI community to develop systems and processes for supporting interactions of human and computer as a team in emergency management 
(Carver and Turoff, 2007). Extreme public concern over pandemics during the past decade suggests that urgent action is required to develop optimal information-sharing environments for promoting good health (Heymann, 2004; Shapiro et al., 2010). Innovative approaches to information flow modeling and analysis for dealing with challenges of coordinating across multilayered agency structures and development of early warnings through social systems using social media analytics may be pivotal to timely response to infectious diseases, which are prevalent in the EU and the US, neighboring regions, and the globe. Network science, digital human modeling combined with user and experience-centered design in HCI offers significant advancement in developing digital interventions for global disease epidemics. For example, network science and computation could be integrated with the work in HCI for emergencies, which advocates with studying and understanding users and engaging users in the design process as well as evaluating designs in a realistic way could influence design and testing of new $\mathrm{HCI}$ design principles within the context of emergencies (Klann et al., 2008), designing context-aware HCI for collaborative emergency management (Flentge et al., 2008), developing digital technologies for supporting, and encouraging public participation of communications during crisis (Landgren and Nulden, 2007; Palen and Liu, 2007).

Biosecurity threats in Australia, US, Europe, and other parts of the world have been recognized as an important area of research due to the connectedness of European borders, which could lead to consequences of spill across other European countries (Sundelius and Grönvall, 2004). However, attempts in dealing with cross borders coordination issues by the 15 member states within the EU Commission suggest the need for institutional preparedness for transnational, rapidly moving diseases which could have deadly consequences (Sundelius and Grönvall, 2004; Lentzos, 2006). Responding effectively to biosecurity can be considered as the heart of national security and is the most fundamental component of the critical infrastructures underpinning social order. Biosecurity response can be conceptualized as interaction network with a flow of information between nodes, each representing an individual actor or agency. Observations of interaction networks in life, engineering, and the physical sciences suggest that key functional properties of these networks are the flow of information they can support, robustness of the flow to node failure, and efficiency of the network. The extreme public concern over pandemics during the past decade suggests that urgent action is required to develop optimal information-sharing environments for the management of health security. Studies in health security highlight the ways in which sharply increased danger of bioterrorism has made infectious diseases a priority in defense and intelligence circles (Heymann, 2003). The challenges of coordinating across multilayered agency structures where innovative approaches to knowledge discovery and decision support informed by dynamic information flow modeling and analysis has been seen as critical importance to the successful management of threats related to biosecurity (Fein, 1991; Aldis, 2008; Chan, 2009). Therefore, coordination for biosecurity needs to be conceptualized as multilayered, involving the orchestration of relationships not only at headquarters but also at the regional, national, and field levels. This can be achieved by integrating the user and experience-centered design in $\mathrm{HCI}$ for developing collective interaction spaces for distributed collaborations ( $\mathrm{Li}, 2010)$.

This will develop biosecurity responsive coordination and recovery systems and provide an understanding of when, how, and where change occur in dynamic environments, and how systems self-organize to coordinate them. The research will quantify and evaluate existing networks of communication and will design new networks for coordinated responses to these outbreaks through a transformation of communication within and between organizations. This research theme addresses an important and timely research dealing with multiorganizational coordinated responses to biosecurity and, in particular, for the case of zoonotic and foodborne outbreaks. The aim is to develop a national and regional level coordinated Biosecurity Networks (BioNet) responses and preparedness for these outbreaks and create a knowledge base for further application of this research in other biosecurity or outbreaks such as foot and mouth disease. In doing so, it will open the door for establishing disaster network science that will significantly influence critical thinking in academic and industrial practices.

\section{Health-Care Service Delivery and Solution Networks}

Health-care service delivery coordination can be investigated by taking a "network" view, which would characterize the study of coordination as interconnected system of nodes (Hossain and Kuti, 2010). User and experience-centered design in HCI can support the flow of information among geographically dispersed community groups and organizations with a potential to make the hierarchical and emerging community networks far more proactive and responsive to the changing circumstances (Tsiknakis et al., 2000). These networks with embedded nodes in the healthcare service delivery and solution may represent anything of the real world from online community of patient network to patient in the intra-hospital network and the organizational networks at different levels who are part of the delivery of service and solution to health and well-being community. Although network structures in organizations can vary greatly (Ahuja et al., 2003), they all maintain one of these foundations: (i) centralized, (ii) decentralized, and (iii) distributed (Baran, 1964). In a centralized structure, all other nodes are directly connected with a single central node, and no link exists among peripheral nodes.

This type of network structure typifies a formal hierarchical network control (Hinds and McGrath, 2006); however, this control is at the expense of creating a single point of failure between the central node and all other boundary nodes (Baran, 1964). Decentralized network structures are able to minimize the problems associated with centralized structure of having a single point of vulnerability by modularizing a large star or centralized network into smaller stars connected with additional links. A distributed network rests on the functions of nodes rather than their interrelationships. As a result, a distributed network provides high level of tolerance for broken links due to the ability to pass network content around via nodes with links still intact (Burt, 2001). Although distributed structures are believed to be very difficult to destabilize, this type of networks is not without its 
flaws. Baran (1964) highlights the redundancy issue of distributed structure, which could potentially inhabit the performance of the distributed network.

Measuring coordination is a challenging task due to difficulties in detecting the type and nature of interactions that exist among actors at different levels, where HCI may offer opportunities for the collection and testing of usability of different pervasive and handhold devices to support coordination in efficient manner (Carroll, 2003). The topology of the network structures described previously assumes symmetry in in-links and out-links; if asymmetries exist, then this introduces a coordination problem since the actors, i.e., service providers, would exhibit far less potential to absorb than to transmit information, means that their signal can "get lost" when seeking to identify concomitant services. For example, in providing effective services for health system, coordination can be measured from how quickly actors (e.g., doctor, health nurses, medical test units, social workers, rehabilitation counselors, clinical psychologist, and pathology department) in the health network exchange information and take immediate actions based on that information for the patients' successful interventions from illness. For health-care organizations, the efficiency and quality of the decisions or actions by social actors in the delivery system would influence the allocation of available resources to care delivery units. Network structure analysis could allow for the systematic evaluation of health operations and its impact of the delivery of effective health-care services.

\section{CONCLUSION}

Disaster network science research can capitalize on open data access and sharing platform in developing trusted recommender

\section{REFERENCES}

Ahuja, M. K., Galletta, D. F., and Carley, K. M. (2003). Individual centrality and performance in virtual R\&D groups: an empirical study. Manage. Sci. 49, 21-38. doi:10.1287/mnsc.49.1.21.12756

Aldis, W. (2008). Health security as a public health concept: a critical analysis. Health Policy Plan. 23, 369-375. doi:10.1093/heapol/czn030

Bandura, A. (1978). Social learning theory of aggression. J. Commun. 28, 12-29. doi:10.1111/j.1460-2466.1978.tb01621.x

Barabási, A. L. (2007). Network medicine - from obesity to the "diseasome". N. Engl. J. Med. 357, 404-407. doi:10.1056/NEJMe078114

Baran, P. (1964). On distributed communications networks. Commun. Syst. IEEE Trans. 12, 1-9. doi:10.1109/TCOM.1964.1088883

Bavelas, A. (1950). Communication patterns in task-oriented groups. J. Acoust. Soc. Am. 22, 725-730. doi:10.1121/1.1906679

Berkman, L. F., Glass, T., Brissette, I., and Seeman, T. E. (2000). From social integration to health: Durkheim in the new millennium. Soc. Sci. Med. 51, 843-857. doi:10.1016/S0277-9536(00)00065-4

Boccaletti, S., Latora, V., Moreno, Y., Chavez, M., and Hwang, D. U. (2006). Complex networks: structure and dynamics. Phys. Rep. 424, 175-308. doi:10.1016/j. physrep.2005.10.009

Borgatti, S. P., Mehra, A., Brass, D. J., and Labianca, G. (2009). Network analysis in the social sciences. Science 323, 892-895. doi:10.1126/science.1165821

Born, C. T., Briggs, S. M., Ciraulo, D. L., Frykberg, E. R., Hammond, J. S., Hirshberg, A., et al. (2007). Disasters and mass casualties: I. General principles of response and management. J. Am. Acad. Orthop. Surg. 15, 388-396. doi:10.5435/00124635-200707000-00004 systems for early warnings of disasters by constructing and validating big data related to usage of social media, approach Government and community-based organizations to improve coordinated response by linking the hierarchical command control interventions to emerging social media based warnings, and develop and evaluate a test bed for just-in-time public programs for emergencies as well as health and well-being education. These activities could be introduced to help shape the future trans-disciplinary approach to disaster network science. Disaster network science can also shape research methods and rigor with a focus on "Network Science," "Complexity Science," or "Dynamical Modeling of Complex Systems and Applications" for developing rigorous research and application platform in the area of disaster preparedness and response. Also, exploring the idea to consider common theoretical and methodological drawn from "Network Science" for theoretical and analytical approach, which have impact on investigating wide variety of phenomenon, i.e., social structures and individuals and group work, financial market systems (crisis), modeling disaster/crisis response in large-scale tsunami, fire, and spread of diseases, such as Ebola. This can further support lab based and experiential as well as experimental work in modeling emerging community network using social medial for detecting early signals from social structures and, therefore, inform hierarchical systems to be more effective in providing robust responses to crisis.

\section{AUTHOR CONTRIBUTIONS}

All authors listed have made substantial, direct, and intellectual contribution to the work and approved it for publication.

Brownstein, J. S., Freifeld, C. C., and Madoff, L. C. (2009). Digital disease detection - harnessing the web for public health surveillance. N. Engl. J. Med. 360, 2153-2157. doi:10.1056/NEJMp0900702

Bruce, C. S. (1999). Workplace experiences of information literacy. Int. J. Info. Manage. 19, 33-47. doi:10.1016/S0268-4012(98)00045-0

Burt, R. S. (2000). The network structure of social capital. Res. Organ. Behav. 22, 345-423. doi:10.1016/S0191-3085(00)22009-1

Burt, R. S. (2001). "Structural holes versus network closure social capital," in Social Capital: Theory and Research, eds N. Lin, K. S. Cook and R. S. Burt, 31-55.

Carrington, P. J., Scott, J., and Wasserman, S. (eds.) (2005). Models and Methods in Social Network Analysis, Vol. 28. Cambridge University Press.

Carroll, J. M. (ed.) (2003). HCI Models, Theories, and Frameworks: Toward a Multidisciplinary Science. New York: Morgan Kaufmann.

Carver, L., and Turoff, M. (2007). Human-computer interaction: the human and computer as a team in emergency management information systems. Commun. ACM 50, 33-38. doi:10.1145/1226736.1226761

Chan, M. (2009). Primary health care as a route to health security. Lancet 373, 1586-1587. doi:10.1016/S0140-6736(09)60003-9

Eisenberg, M. B., Lowe, C. A., and Spitzer, K. L. (2004). Information Literacy: Essential Skills for the Information Age. Westport, CT: Greenwood Publishing Group.

Fein, R. (1991). The health security partnership. JAMA 265, 2555-2558. doi:10.1001/jama.1991.03460190133035

Flentge, F., Weber, S. G., Behring, A., and Ziegert, T. (2008). "Designing context-aware HCI for collaborative emergency management," in Int'l Workshop on HCI for Emergencies in Conjunction with CHI, Vol. 8, Florence.

Friedkin, N. E. (1998). A Structural Theory of Social Influence. Cambridge: Cambridge University Press. 
Ginsberg, J., Mohebbi, M. H., Patel, R. S., Brammer, L., Smolinski, M. S., and Brilliant, L. (2009). Detecting influenza epidemics using search engine query data. Nature 457, 1012-1015. doi:10.1038/nature07634

Granovetter, M. S. (1973). The strength of weak ties. Am. J. Sociol. 78, 1360-1380. doi:10.1086/225469

Hanish, L. D., and Rodkin, P. C. (2007). Bridging children's social development and social network analysis. New Dir. Child Adolesc. Dev. 2007, 1-8. doi:10.1002/ cd. 197

Heymann, D. (2003). The evolving infectious disease threat: implications for national and global security. J. Hum. Dev. 4, 191-207. doi:10.1080/14649880 32000087541

Heymann, D. L. (2004). The international response to the outbreak of SARS in 2003. Philos. Trans. R. Soc. Lond. B Biol. Sci. 359, 1127-1129. doi:10.1098/ rstb.2004.1484

Hinds, P., and McGrath, C. (2006). "Structures that work: social structure, work structure and coordination ease in geographically distributed teams," in Proceedings of the 2006 20th Anniversary Conference on Computer Supported Cooperative Work (ACM), Alberta 343-352.

Hossain, L., and Kuti, M. (2010). Disaster response preparedness coordination through social networks. Disasters 34, 755-786. doi:10.1111/j.1467-7717.2010. 01168.x

Kalichman, S. C., Benotsch, E. G., Weinhardt, L., Austin, J., Luke, W., and Cherry, C. (2003). Health-related Internet use, coping, social support, and health indicators in people living with HIV/AIDS: preliminary results from a community survey. Health Psychol. 22, 111. doi:10.1037/0278-6133.22.1.111

Klann, M., Malizia, A., Chittaro, L., Aedo Cuevas, I., and Levialdi, S. (2008). "HCI for emergencies," in CHI'08 Extended Abstracts on Human Factors in Computing Systems (ACM), Florence, 3945-3948.

Krackhardt, D. (1992). The strength of strong ties: the importance of philos in organizations. Netw. Organ. Struct. Form Action 216, 239.

Landgren, J., and Nulden, U. (2007). "A study of emergency response work: patterns of mobile phone interaction," in Proceedings of the SIGCHI Conference on Human Factors in Computing Systems (ACM), San Jose, 1323-1332.

Lentzos, F. (2006). Rationality, risk and response: a research agenda for bio-security. Biosocieties 1, 453. doi:10.1017/S1745855206004066

Li, J. (2010). "Designing interaction spaces for distributed collaborations," in Proceedings of the 22nd Conference of the Computer-Human Interaction Special Interest Group of Australia on Computer-Human Interaction (ACM), Brisbane, 432-434.

Lindley, S. E., Banks, R., Harper, R., Jain, A., Regan, T., Sellen, A., et al. (2009). Resilience in the face of innovation: household trials with BubbleBoard. Int. J. Hum. Comput. Stud. 67, 154-164. doi:10.1016/j.ijhcs.2008.07.008

Marsden, P. V., and Friedkin, N. E. (1993). Network studies of social influence. Sociol. Methods Res. 22, 127-151. doi:10.1177/0049124193022001006

Palen, L., and Liu, S. B. (2007). "Citizen communications in crisis: anticipating a future of ICT-supported public participation," in Proceedings of the SIGCHI Conference on Human Factors in Computing Systems (ACM), San Jose, 727-736.

Pentland, A., Choudhury, T., Eagle, N., and Singh, P. (2005). Human dynamics: computation for organizations. Pattern Recognit. Lett. 26, 503-511. doi:10.1016/j.patrec.2004.08.012

Sadilek, A., Kautz, H., and Silenzio, V. (2012). "Modeling spread of disease from social interactions," in Proceedings of the Sixth International AAAI Conference on Weblogs and Social Media (Association for the Advancement of Artificial Intelligence), Dublin, 322-329.

Shapiro, J. S., Genes, N., Kuperman, G., Chason, K., Clinical Advisory Committee H1N1 Working Group, New York Clinical Information Exchange, and Richardson, L. D. (2010). Health information exchange, biosurveillance efforts, and emergency department crowding during the spring 2009 H1N1 outbreak in New York City. Ann. Emerg. Med. 55, 274-279. doi:10.1016/j. annemergmed.2009.11.026

Streefkerk, J. W., Neef, M., Meesters, K., Pieneman, R., and Dongen, K. V. (2014). "HCI challenges for community-based disaster recovery," in 5th International Conference on Digital Human Modeling and Applications in Health, Safety, Ergonomics and Risk Management, DHM 2014-Held as Part of 16th International Conference on Human-Computer Interaction, HCI International 2014, 8529 LNCS (Heraklion: Springer-Verlag), 637-648.

Sundelius, B., and Grönvall, J. (2004). Strategic dilemmas of biosecurity in the European Union. Biosecurity Bioterrorism 2, 17-23. doi:10.1089/153871304322964309

Taleb, N. (2005). The Black Swan: Why Don't We Learn that We Don't Learn. New York: Random House.

Thomas, J., and Wong, P. C. (2004). Visual analytics. IEEE Comput. Graph. Appl. 24, 20-21. doi:10.1109/MCG.2004.39

Tsiknakis, M., Katehakis, D. G., and Orphanoudakis, S. C. (2000). "Information infrastructure for an integrated healthcare services network," in Proceedings IEEE EMBS International Conference on Information Technology Applications in Biomedicine (Virginia: IEEE), 278-283.

Turner, J. H. (1988). A Theory of Social Interaction. Stanford University Press, California.

Wasserman, S., and Faust, K. (1994). Social Network Analysis: Methods and Applications. Cambridge: Cambridge University Press.

Watts, J. D. (2004). The "new" science of networks. Annu. Rev. Sociol. 30, 243-270. doi:10.1146/annurev.soc.30.020404.104342

Wellman, B. (1988). "Structural analysis: from method and metaphor to theory and substance," in Social Structures: A Network Approach, eds B. Wellman and S. D. Berkowitz (Cambridge: Cambridge University Press), 19-61.

Wish, M., Deutsch, M., and Kaplan, S. J. (1976). Perceived dimensions of interpersonal relations. J. Pers. Soc. Psychol. 33, 409. doi:10.1037/0022-3514.33.4.409

York, J., and Pendharkar, P. C. (2004). Human-computer interaction issues for mobile computing in a variable work context. Int. J. Hum. Comput. Stud. 60, 771-797. doi:10.1016/j.ijhcs.2003.07.004

Conflict of Interest Statement: The authors declare that the research was conducted in the absence of any commercial or financial relationships that could be construed as a potential conflict of interest.

Copyright () 2016 Hossain and Feng. This is an open-access article distributed under the terms of the Creative Commons Attribution License (CC BY). The use, distribution or reproduction in other forums is permitted, provided the original author(s) or licensor are credited and that the original publication in this journal is cited, in accordance with accepted academic practice. No use, distribution or reproduction is permitted which does not comply with these terms. 